\title{
Distal Interphalangeal Joint 2
}

National Cancer Institute

\section{Source}

National Cancer Institute. Distal Interphalangeal Joint 2. NCI Thesaurus. Code C102291.

A ginglymoid (hinge) synovial joint within the second digit of the hand or foot connecting the middle and distal phalanges. 